\title{
Shock Dynamics in the Sub-Nanometer Femtosecond Domain*
}

\author{
A. M. KARO and T. M. DEBONI \\ Lawrence Livermore National Laboratory, Livermore, California \\ J. R. HARDY \\ University of Nebraska, Lincoln, Nebraska \\ G. A. WEISS \\ University of California, Berkeley, California
}

\begin{abstract}
New experimental techniques combined with computer molecular dynamics simulations of increasing complexity are beginning to permit a detailed examination of the initial steps in the complex chemistry describing the high-temperature, high-pressure regime associated with combustion and detonation as well as processes that may be involved in shock-induced chemistry. We present the results of several simulations that describe the generation of moderately strong shocks and their interaction with molecules embedded in weakly-bound clusters, with material defects such as microscopic voids, and with molecular species placed in the neighborhood of such defects.
\end{abstract}

\section{Introduction}

In our original series of studies [1,2] we found that shocks in regular one-dimensional and two-dimensional lattices preserved their integrity to a very high degree, were of atomic dimensions, and because of this were able to deposit their energy selectively to the edge of the system when reaching a lattice boundary, producing small, energetic spalled fragments. This early work was stimulated by the suggestion of Walker and Wasley [3] that shocks could break chemical bonds mechanically and athermally. It also coincided with the beginning of today's immense range of studies on nonlinear systems and appears to have been the first in which the consequences of this "soliton-like" behavior were described as being important for a full understanding of the mechanisms underlying the shock-induced initiation of energetic materials.

In our calculations we found the situation in the shock front to be so completely nonergodic that the concepts of equilibrium thermodynamics and statistical mechanics would be unlikely to have meaning in this region. Thus, in a regular lattice

* Work performed in part under the auspices of the U.S. Department of Energy by the Lawrence Livermore National Laboratory under contract number W-7405-ENG-48 and under the auspices of the Office of Naval Research. 


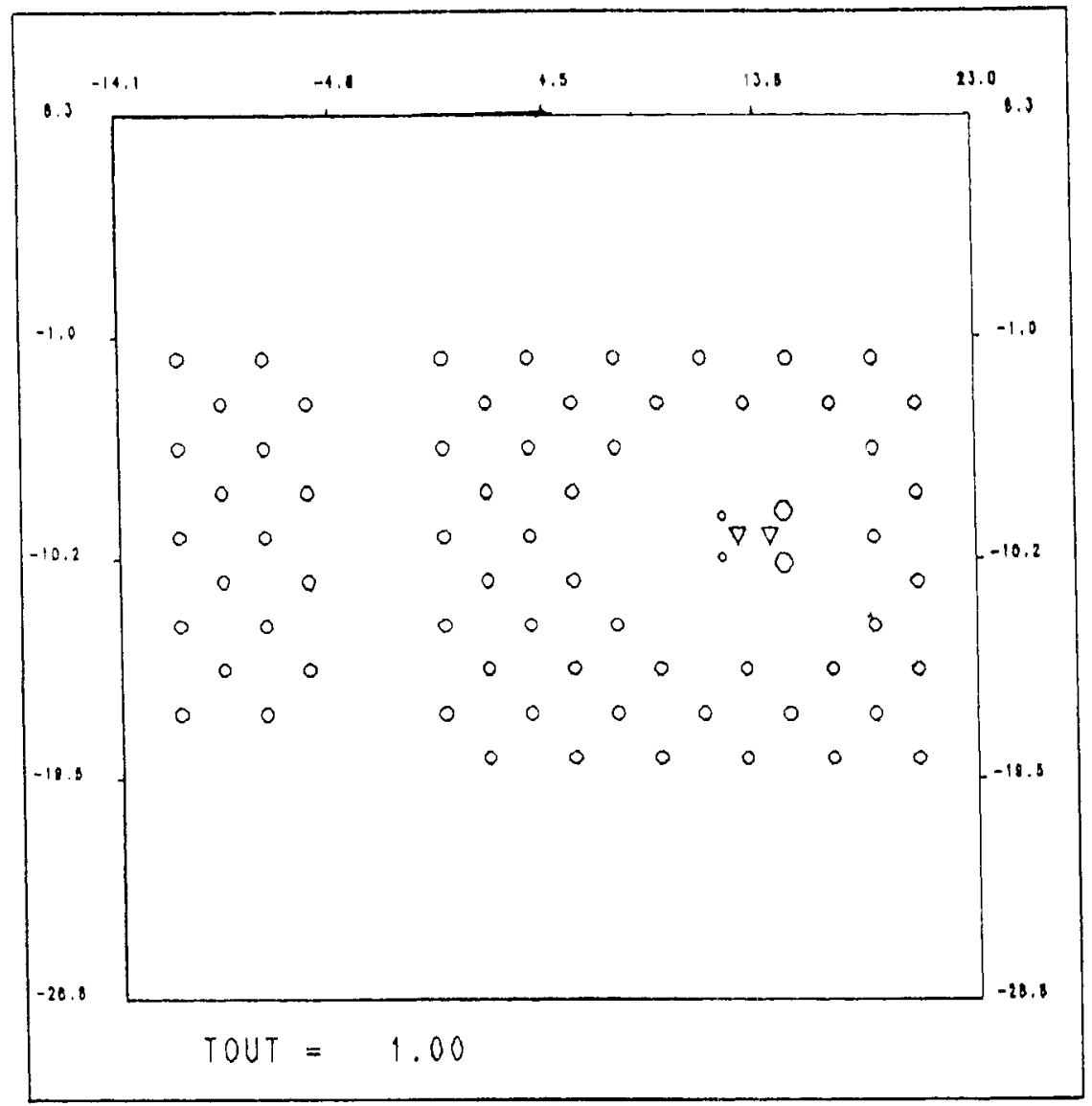

Figure 1. The nitromethyl radical placed inside a void located within a weakly-bound host lattice. The times, denoted by TOUT, are in units of $10^{-14} \mathrm{~s}$. The $\mathrm{H}$ atoms are shown as small circles, $\mathrm{C}$ and $\mathrm{N}$ atoms are denoted by triangles, and $\mathrm{O}$ atoms by large circles. At $0.01 \mathrm{ps}$ the plate is in motion toward the lattice at a velocity of $50 \AA / \mathrm{ps}$. At 0.36 ps the void has almost completely collapsed around the molecule, and at $0.80 \mathrm{ps}$ the lattice has vaporized.

or a uniform liquid the front was found to be a few angstroms in width propagating with a velocity in the range of 2 to $5 \mathrm{~mm} / \mu \mathrm{s}$ or 2 to $5 \AA$ in $10^{-13} \mathrm{~s}$. Time scales of this magnitude correlate closely with the range of vibrational frequencies associated with strong covalent bonds, for example, from ca. $4400 \mathrm{~cm}^{-1}$ for $\mathrm{H}_{2}$ down to less than $1000 \mathrm{~cm}^{-1}$ for vibrational modes of polyatomic organic systems (corresponding to frequencies ranging from $13 \times 10^{13} \mathrm{sec}^{-1}$ to less than $\left.3 \times 10^{13} \mathrm{sec}^{-1}\right)$. Comparable periods of vibration will be from 8 to more than $40 \mathrm{fs}$; during this time, at a velocity of $5 \mathrm{~mm} / \mu \mathrm{s}$, a shock front will have advanced from 0.4 to $2.0 \AA$. Thus, thermal lattice vibrations and low-lying intramolecular modes, as well as weak intermolecular modes, are essentially spatially frozen with respect to the subpicosecond amplitude 


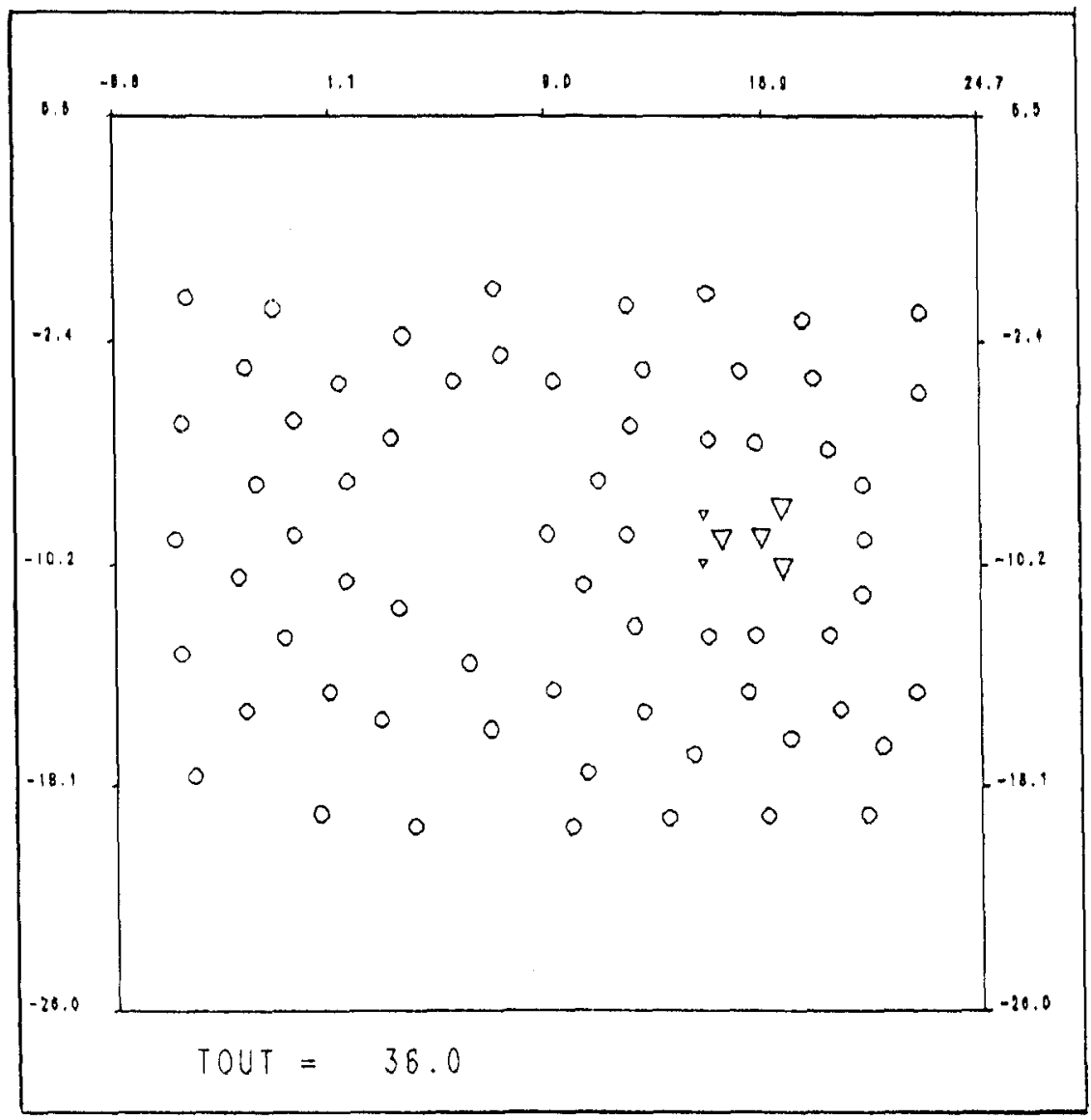

Figure 1. (Continued)

rise time of the advancing shock front. This effectively decouples the shock front translational motion from lattice thermal motion and results in a lack of local thermodynamic equilibrium [4,5].

Following this early work on simple monatomic and diatomic systems, a large number of computer simulations of shocks passing through both liquids and solids have shown not only that the energy localized in the region of the shock front can impart to surface atoms sufficient momentum to cause spall, but also that the front can deposit to defect regions within the bulk sufficient energy to disrupt the lattice or to cause bond rearrangement [6-9].

Our recent studies [10-12] have focused on the microscopic details of energy transfer in shocked crystal and molecular lattices. The molecular units forming a molecular lattice and the various types of point, line, and volume defects all represent local inhomogeneities which can act to transfer the energy from the shock front to the lattice. From a study of the dynamics of energy exchange, we obtain insight 


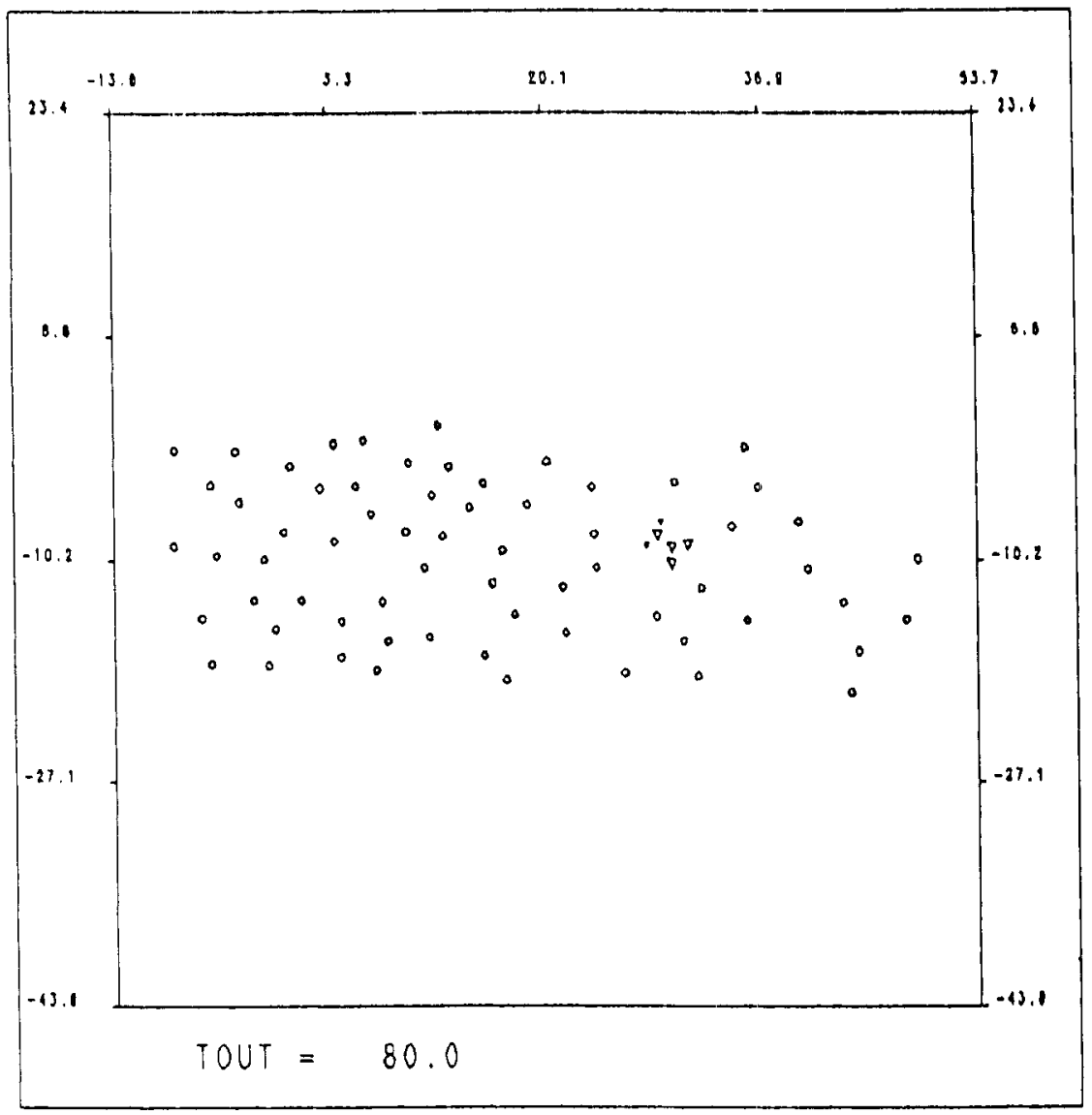

Figure 1. (Continued)

concerning the rate of energy concentration and partitioning in molecular bonds, as well as an understanding of the role of each of the various types of defects in focusing the energy from the shock front into these microscopic "hot spots."

Experimental techniques have recently begun to address the subnanometer, femtosecond regime long explored by computer simulation [13-17]. To complement such experiments we have initiated simulations that parallel these experiments; for example, we have begun a series of simulations of the energetic impact of molecules and clusters on solid surfaces, including the fragmentation that often occurs after the surface collision.

In the following sections we will describe two recent simulations: the first addresses the complex situation in which a propagating shock front interacts with a void within which a molecular unit has been placed. Here we have the interplay between fast shock rise times and an extreme impedance mismatch via the masses and interactions between host lattice, void, and molecule, where both strong and weak 
TABLE I. Parameters for the Morse and Lennard-Jones potentials used in the simulation of a nitromethyl radical placed within a void located inside a host lattice of atoms M. Morse: $V(r)=D_{e}\left[e^{-2 \beta\left(r-r_{e}\right)}-\right.$ $\left.2 e^{-\beta\left(r-r_{e}\right)}\right]$; Lennard-Jones: $V(r)=4 D_{e}\left[(\sigma / r)^{12}-(\sigma / r)^{6}\right]$. The last four potentials were chosen to prevent undue distortion of the molecule under shock loading.

\begin{tabular}{|c|c|c|c|c|c|c|c|}
\hline Bond & $D_{e}(\mathrm{eV})$ & $r_{e}(\AA)$ & $\beta\left(\AA^{-1}\right)$ & $\omega_{e}\left(\mathrm{~cm}^{-1}\right)$ & $D_{e}(\mathrm{eV})$ & $r_{e}(\AA)$ & $\sigma(\AA)$ \\
\hline & \multicolumn{4}{|c|}{ Morse potentials } & \multicolumn{3}{|c|}{ Lennard-Jones potentials } \\
\hline MM & 0.268 & 2.633 & 2.000 & 139.5 & 0.020 & 2.633 & 2.345 \\
\hline MH & 0.268 & 2.633 & 2.000 & 770.5 & 0.020 & 3.100 & 2.762 \\
\hline MC & 0.268 & 2.633 & 2.000 & 241.7 & 0.020 & 4.190 & 3.733 \\
\hline MN & 0.268 & 3.723 & 2.000 & 226.9 & 0.020 & 4.320 & 3.849 \\
\hline MO & 0.268 & 3.723 & 2.000 & 215.0 & 0.020 & 3.100 & 2.762 \\
\hline $\mathrm{CH}$ & 3.640 & 1.090 & 2.094 & 3066.0 & & & \\
\hline $\mathrm{CN}$ & 2.557 & 1.390 & 3.126 & 1450.1 & & & \\
\hline NO & 6.614 & 1.220 & 1.869 & 1296.9 & & & \\
\hline OO & 1.000 & 2.140 & 2.397 & 625.0 & & & \\
\hline $\mathrm{HH}$ & 1.000 & 1.810 & 1.396 & 1455.7 & & & \\
\hline $\mathrm{HN}$ & 1.000 & 2.190 & 0.936 & 714.6 & & & \\
\hline $\mathrm{CO}$ & 1.000 & 2.240 & 1.722 & 485.0 & & & \\
\hline $\mathrm{HO}_{\text {cis }}$ & 0.500 & 2.590 & 0.906 & 486.8 & & & \\
\hline $\mathrm{HO}_{\text {trns }}$ & 0.500 & 3.250 & 1.953 & 1049.8 & & & \\
\hline
\end{tabular}

intra- and intermolecular forces are present. In the second simulation we consider the multiple interactions present as a weakly-bonded cluster containing a covalentlybonded species impacts a wall. This generates a micro-shock traveling back through the cluster that can interact with the molecular species before the main moleculewall collision occurs. Such cluster wall collisions have been proposed as a means of investigating experimentally primary unimolecular decomposition processes and collision-induced decomposition in the subnanometer, femtosecond regime [18].

\section{Simulations}

The basic method of computer molecular dynamics, specifically as applied to the study of fast transient processes in condensed systems, has been described before in detail [5] and need not be repeated here. Briefly, it enables the monitoring, by computer solution of Newton's second law equations of motion for the individual atoms, of the time evolution of the system under study. The set of coupled nonlinear, second-order differential equations that represent the forces are reduced to a doubled set of first-order ordinary differential equations by standard procedures. The initial positions and velocities of the particles represent the initial conditions on these equations. For the numerical solution of this set of equations we have used the variable-step, variable-order, implicit Adams' method with functional (or fixed point) iteration; a generalization of the classical Adams-Bashforth-Moulton method [19]. Relative error build-up is controlled by an error tolerance parameter, so that numerical error is typically kept to less than $10^{-6}$ for times up to 10 to 50 picoseconds during the simulation. 


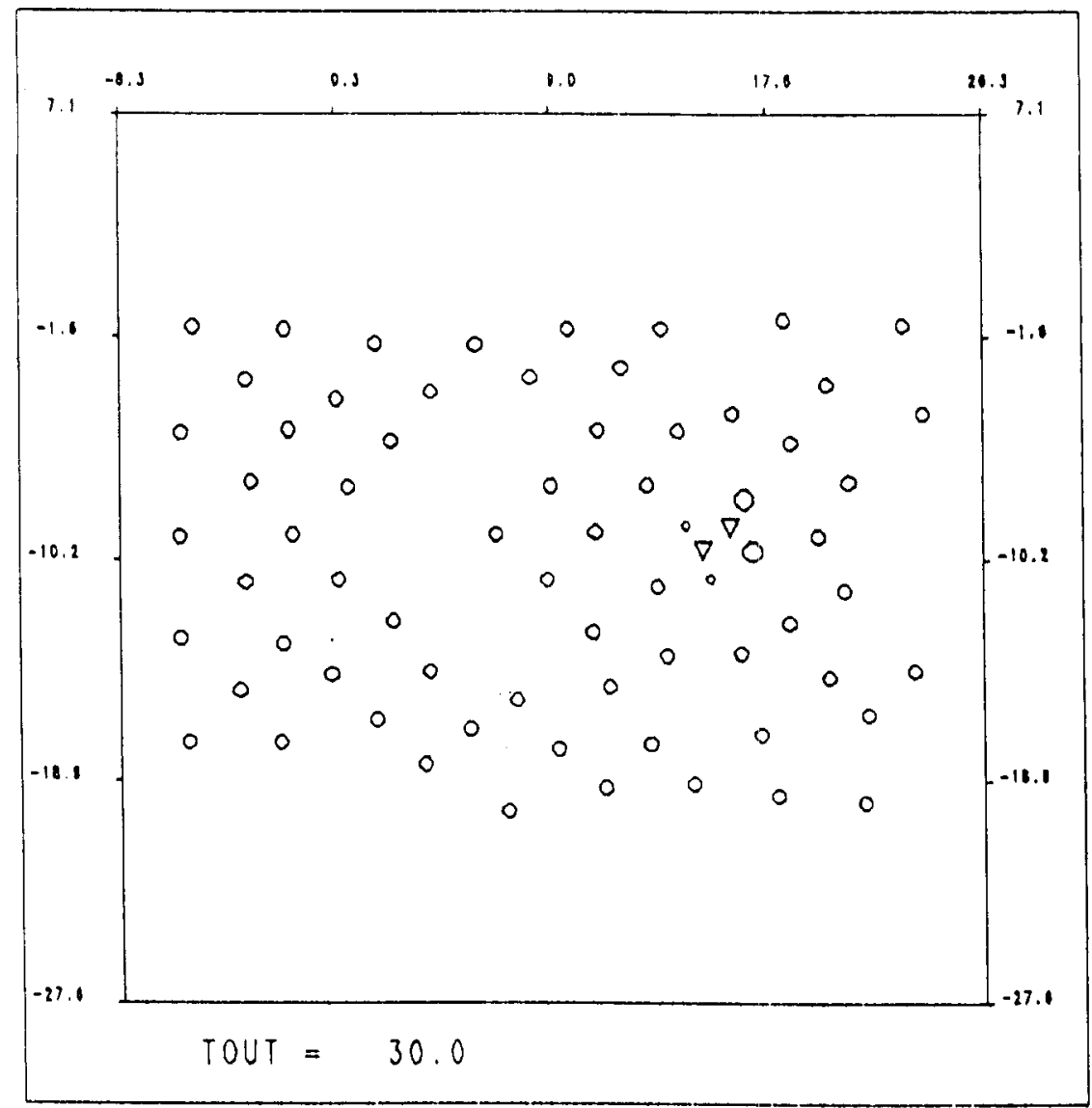

Figure 2. The nitromethyl radical placed inside a void located within the more stronglybound host lattice. The initial configuration is the same as that shown in Figure 1. Comparing results at 0.30 and $0.36 \mathrm{ps}$ we can see the violent distortion and twisting of the molecule. At $0.80 \mathrm{ps}$ the lattice is rapidly disassembling, however the new void formed during the shock sequence continues to persist.

\section{Shock Interaction with a Micro-void Plus Molecule}

We have shown that a molecule well-embedded in a host matrix or in a structurally perfect molecular crystal will rapidly ( $<0.1 \mathrm{ps}$ ) take up energy from the shock front [10-12]. If the surrounding cage remains intact, this energy flows back into the lattice just as rapidly. Molecules on or near the surface can spall along with the rapidly-disassembling cage, remaining in energetically excited states.

Within a real composite explosive, voids of various sizes are present, ranging from 10's of $\AA$ to 10 's of $\mu$. It is well known that the sensitivity of an energetic material is very directly correlated with the type and number of defects such as voids. To gain some insight into the dynamics of the energy flux in the region of a 


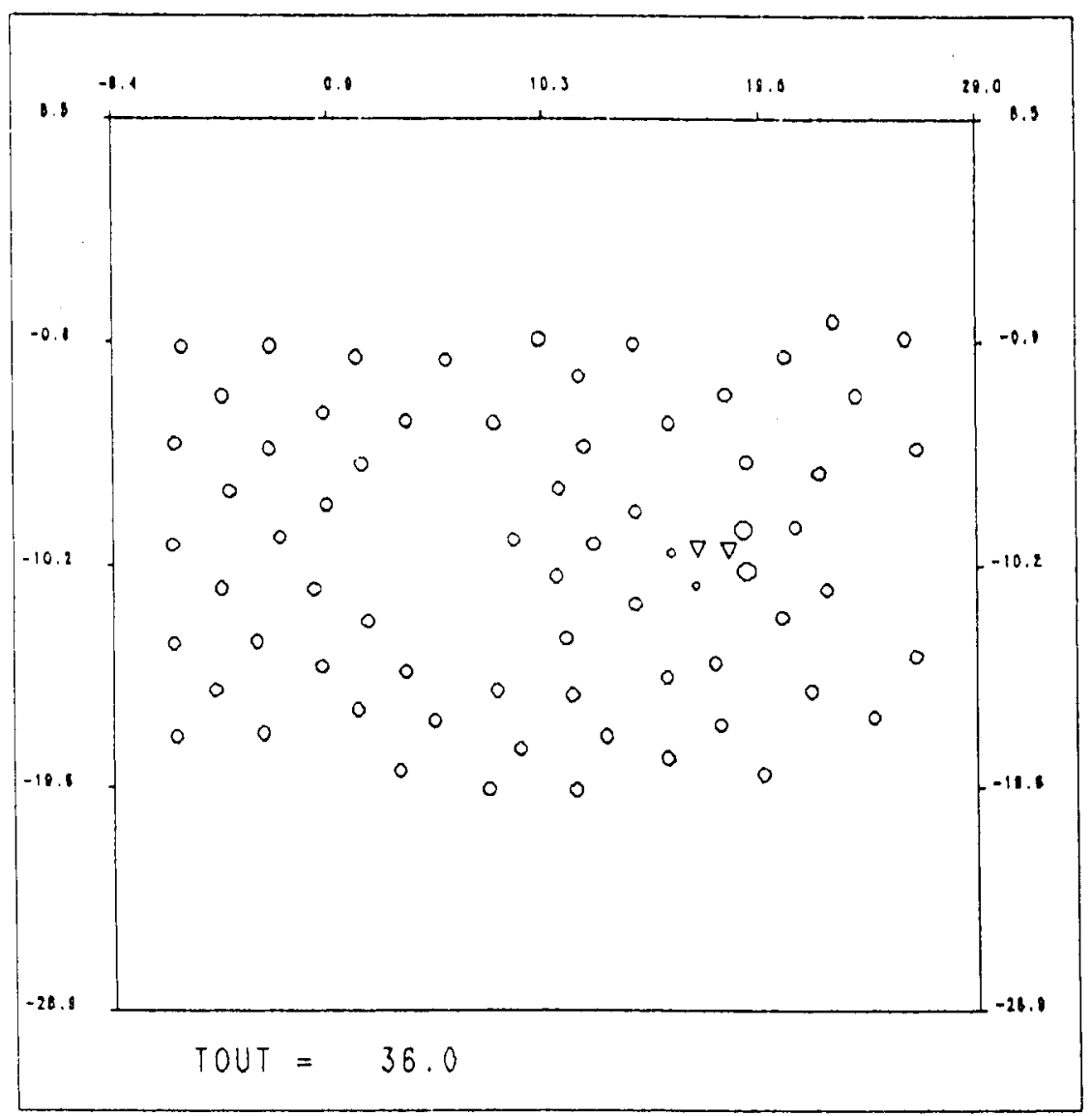

Figure 2. (Continued)

microvoid after passage of a shock front, as well as insight into the nature of the subsequent energy transfer to a tightly bound molecular unit, we formed a void inside a host lattice, within which we placed a nitromethyl unit. This is shown in Figure 1(a). To initiate the shock a section of lattice, or "plate," was set in motion toward the main structure at a velocity which would create a shock in the vicinity of $100 \mathrm{kbar}(10 \mathrm{GPa}$ ). To investigate the importance of impedance matching (via the cage) two lattices were considered, one with very weak binding energies $\approx 0.02$ $\mathrm{eV}$, thus simulating a rare gas matrix, and one with binding energies $\approx 0.27 \mathrm{eV}$, in the range of a more strongly-bound molecular crystal. The heavier masses of the atomic units forming the host lattice also present a mismatch between the host lattice and the individual atoms forming the nitromethyl unit. The parameters defining the potentials, masses, and configuration of the system are given in Table I. It can be seen that the masses of the host particles and their interaction with the void and the molecule contained therein and with other host particles simulate to 


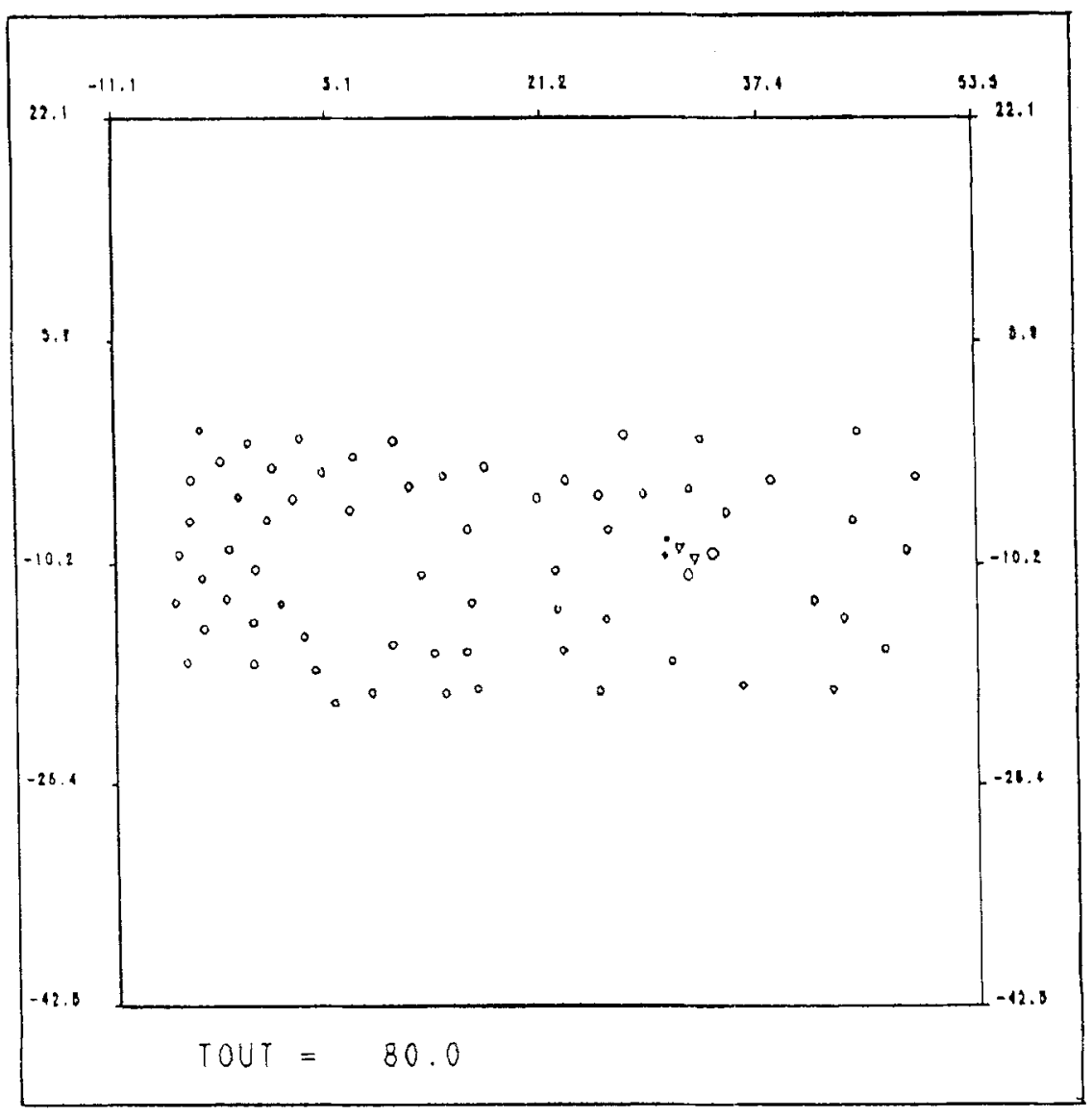

Figure 2. (Continued)

some extent a nitromethyl lattice containing a void, including a detailed probe of the energy flux into and out of a representative molecular unit within the void. Figures 1 (b) and 1 (c) show results after 0.36 and 0.80 ps for the first case, and Figures 2(a), 2(b), and 2(c) show the time evolution after $0.30,0.36$, and $0.80 \mathrm{ps}$ for the second case. We note that void collapse around the molecular unit occurs in less than 0.5 ps with a void reforming to the left of the molecule, now enclosed by a combination of jetting material from the anterior wall and void collapse. Reappearance of voids ("cold spots") has been suggested [20] and would be due to relaxation of the medium from the extreme overdriven regime in the shock front. In Figures 3 and 4 we show the "caged" vibrational energy, internal energy, and total energy (vibrational + rotational + center-of-mass) taken up by the nitromethyl radical for both cases. The term "caged" refers to adding to the kinetic energies only the intramolecular potential energy of the molecular unit. Including the potential energy between the molecule and the cage gives the "uncaged" energies 

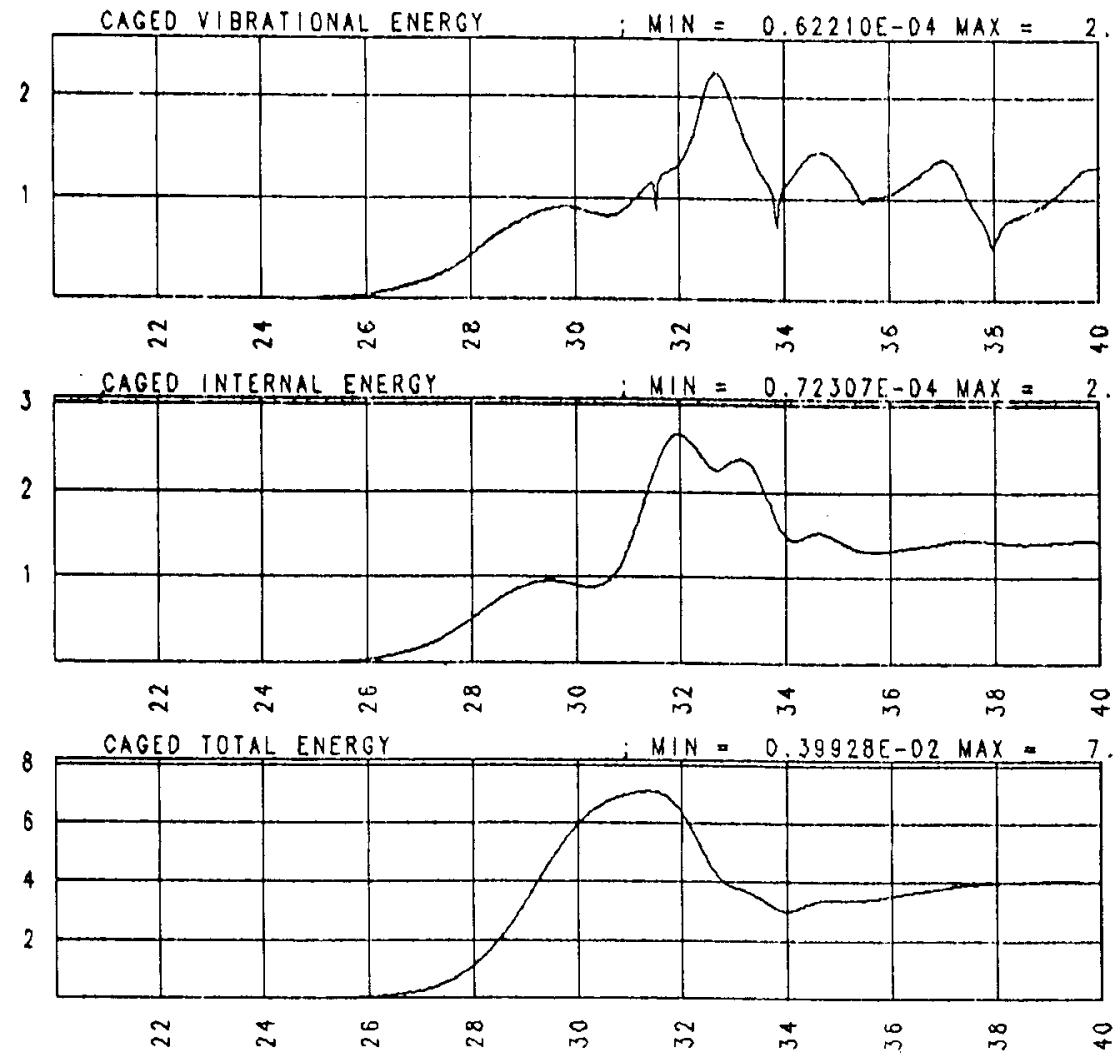

Figure 3. The vibrational, internal, and total energy (in $\mathrm{eV}$ ) of the nitromethyl radical as a function of time (in units of $10^{-14} \mathrm{~s}$ ) for the weakly-bound case described in the text and shown in Figure 1.

which we have also monitored as a function of time. The initial uptake of energy occurs in $\approx 30$ to $40 \mathrm{fs}$. For the more strongly-bound system there is partial decoupling of the lattice energy and the energy of the molecule, with the total energy of the molecular unit at $0.80 \mathrm{ps}$ around $8 \mathrm{eV}$ and the average host kinetic energy around $1 \mathrm{eV}$.

For the weakly-bound system, the results are similar with about $7 \mathrm{eV}$ of total energy taken up in $\approx 35 \mathrm{fs}$, and with a gradual loss of the total energy to $\approx 4 \mathrm{eV}$ at $0.80 \mathrm{ps}$, as the c.o.m. energy drops from $3.7 \mathrm{eV}$ to $2 \mathrm{eV}$ over the last $0.2 \mathrm{ps}$. The average lattice kinetic energy remains $\approx 0.5 \mathrm{eV}$ at the end of the simulation. In monitoring the internal energy as a function of time for the $\mathrm{CN}$ bond, we find a maximum value of $3.0 \mathrm{eV}$ for the more strongly-bound lattice and $0.92 \mathrm{eV}$ for the weakly-bound lattice. Considering the overall extreme impedance mismatch caused by the void and the weak coupling of host lattice to the molecule, these are rather remarkable results, showing that the initial very strong energy transfer occurring in moderate to strong shocks must be taken into consideration before the longer 

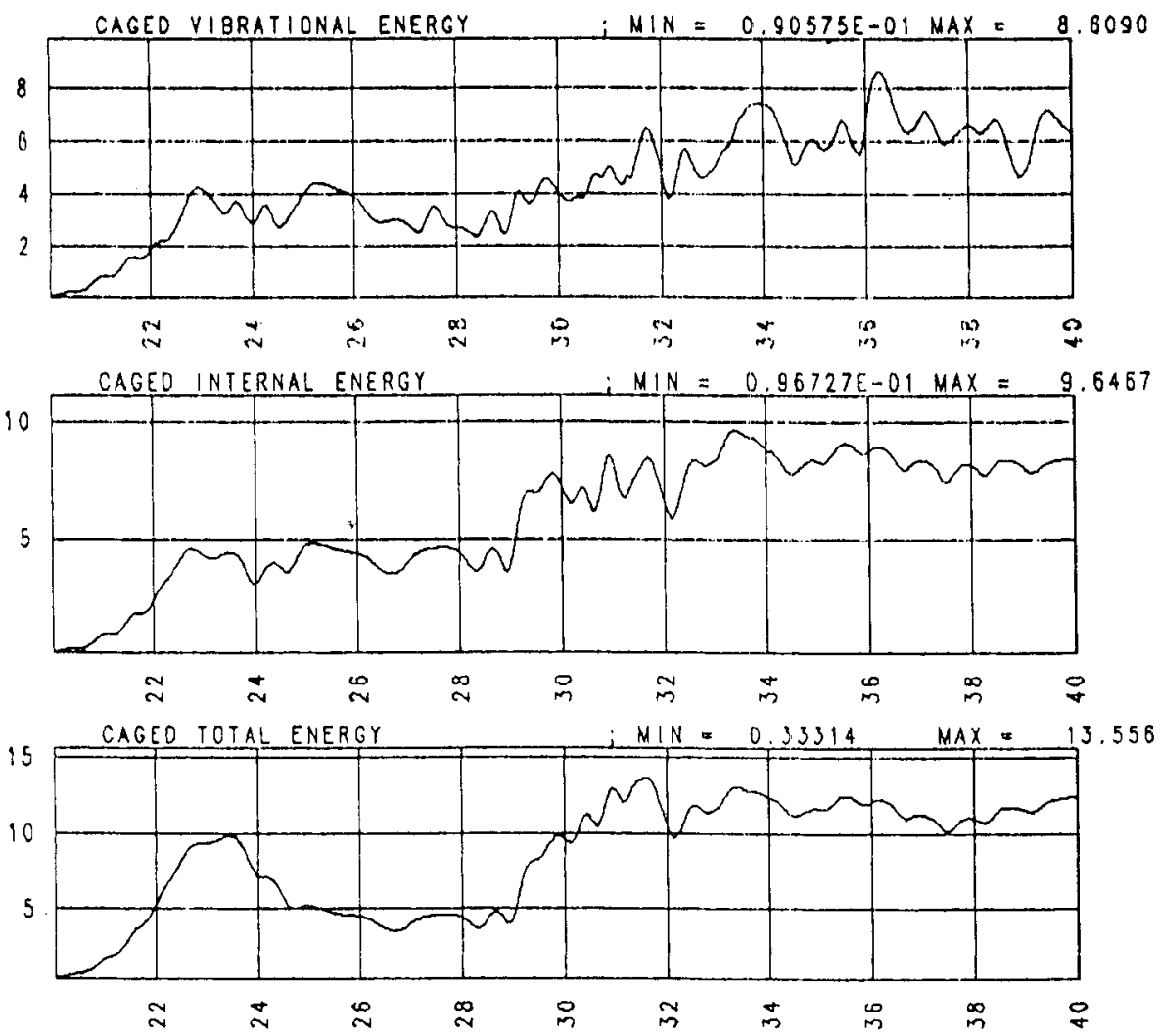

Figure 4. The vibrational, internal, and total energy (in $\mathrm{eV}$ ) of the nitromethyl radical as a function of time (in units of $10^{-14} \mathrm{~s}$ ) for the more strongly-bound case described in the text and shown in Figure 2.

time mechanisms of "vibrational up-pumping" that have been postulated [21] begin to take effect.

\section{Cluster-Wall Impacts}

Experimental techniques for forming a supersonic stream of clusters of atoms and molecules with laboratory-frame kinetic energies in the range of 1 to $10 \mathrm{eV}$ are now well-developed [17]. Embedding a fragment or molecule by placing it within a cluster will give rise to a dynamic sequence in which there would first be shock compression and excitation of the fragment as a micro-shock travels back from the forward part of the cluster after initial contact with the wall is made, followed by rapid disassembly of the weakly-bound cluster and further collisioninduced dissociation as the molecular fragment itself collides with the wall. To shed some light on to what extent such a complex sequence could be followed experimentally, we have simulated the simple case of an $\mathrm{HI}$ diatomic molecule embedded 
TABLE II. Parameters for the Morse and Lennard-Jones potentials used in the simulation of an HI molecule placed within an argon cluster directed toward a heavy-atom wall (W). Morse: $V(r)=$ $D_{e}\left[e^{-2 \beta\left(r-r_{e}\right)}-2 e^{-\beta\left(r-r_{e}\right)}\right]$; Lennard-Jones: $V(r)=4 D_{e}\left[(\sigma / r)^{12}-(\sigma / r)^{6}\right]$. Parameters for the wall give a tightly-bound structure to which the incoming cluster particles can only physisorb. Values in square brackets are chosen so that, excluding HI, only weak binding occurs in the rest of the system.

\begin{tabular}{lccccc}
\hline \hline Bond & $D_{e}(\mathrm{eV})$ & $r_{e}(\AA)$ & $\beta\left(\AA^{-1}\right)$ & $\omega_{e}\left(\mathrm{~cm}^{-1}\right)$ & $\sigma(\AA)$ \\
\hline WW & 4.772 & 1.000 & 1.93 & 136.9 & - \\
HI & 3.196 & 1.610 & 1.75 & 2309.0 & - \\
ArAr & 0.012 & 3.760 & - & 25.7 & 3.350 \\
ArH & 0.004 & 3.570 & - & - & 3.181 \\
ArI & {$[0.050]$} & {$[4.000]$} & - & - & 3.564 \\
WAr & {$[0.004]$} & {$[3.570]$} & - & - & 3.181 \\
WH & {$[0.004]$} & {$[3.570]$} & - & - & 3.181 \\
WI & {$[0.050]$} & {$[4.000]$} & - & &
\end{tabular}

in an argon cluster, directed toward a heavy inert wall, at a translational energy of $\approx 5 \mathrm{eV} / \mathrm{Ar}$ atom. The parameters defining the potentials and the configuration of the wall and cluster are given in Table II. Figure 5 shows schematically a typical result from a series of calculations in which the relative orientation of the HI molecule with respect to the wall was varied. In the figure the molecule remains intact, but the cluster has vaporized. We have found that molecular dissociation is orientation-dependent and that vibrational excitation of a surviving molecule can be large. Future studies in this area would involve more complex molecules and molecular fragments.

\section{Conclusions}

The results of simulating a shock interacting with a complex heterogeneity (represented in the present instance by a molecule located within a void) show, first, that the basic situation remains the same as found in our earlier model studies [912]: transfer of energy from a moderately-strong shock front occurs rapidly on time scales of femtoseconds, with the resulting internal energy of the region far larger than the thermal energy of the host lattice. This is true both for extremely weakly-bound rare gas host lattices and for lattices with binding more appropriate to that found for molecular crystals. Complex dynamic processes are involved, as illustrated by following the center-of-mass energy of the enclosed molecule which, for the weakly-bound system, first rises to $\approx 6 \mathrm{eV}$ in $40 \mathrm{fs}$ as jetting material from the front wall of the void impacts and accelerates the molecule, then falls to $<1$ $\mathrm{eV}$ in 25 fs as the molecule impacts the "soft" far side of the void [22]. The c.o.m. energy then rises more slowly to around $3.5 \mathrm{eV}$ as the molecule and the host material are driven forward by the momentum imparted by the impacting plate. Similar results occur for the case where the host lattice is more strongly bound, with a rise time of the initial c.o.m. energy to $8 \mathrm{eV}$ in $30 \mathrm{fs}$, then an abrupt drop within $20 \mathrm{fs}$ to $<0.1 \mathrm{eV}$ followed by an increase in the c.o.m. energy as the whole system is 


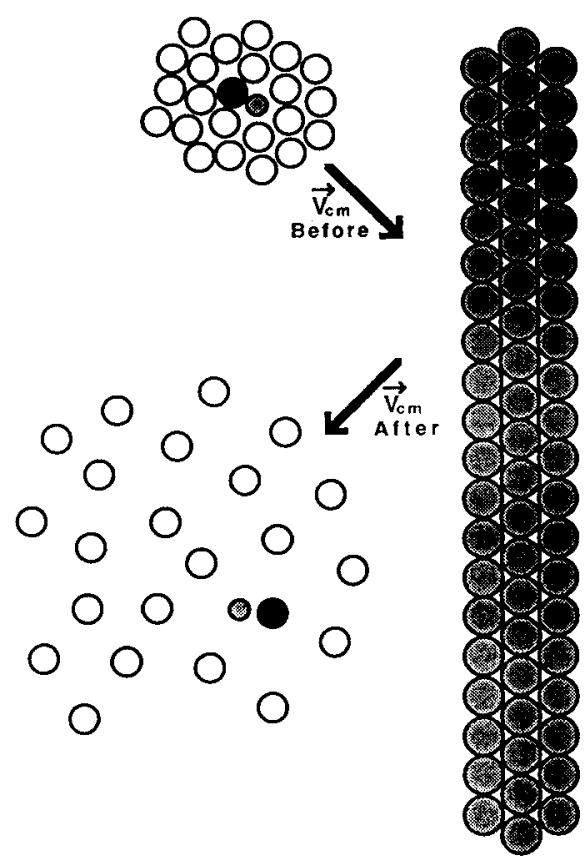

Figure 5. A schematic representation of an argon cluster, with a translational kinetic energy of $5 \mathrm{eV} / \mathrm{Ar}$ atom, containing an $\mathrm{HI}$ molecule, before and after impacting a heavy wall. The results of several calculations indicate that when the orientation of the HI molecule is such that the $\mathrm{H}$ atom collides with the wall first, dissociation occurs, whereas if the I atom is first to hit, the molecule remains intact and vibrotationally excited. At this translational energy the rare gas cluster rapidly vaporizes.

driven forward by the plate [22]. With a larger host-lattice ensemble, which would more realistically represent an experimental situation, the subsequent rise of c.o.m. energy would not be expected to occur to any marked degree; however, the initial vibrational, rotational, and translational shock-up of the molecule would occur, followed by transfer of c.o.m. translational energy to additional vibrational and rotational motion of the molecule and to heating the back wall of the void itself. Thus, because of the presence of voids there are additional dynamic processes that facilitate the energy transfer from a shock front, features that would be expected to aid the initial endothermic decomposition steps in the shock initiation of an energetic system, with the excited fragments and the energetically excited region around the void forming the basis for ensuing exothermic reactions.

Similarly, a cladded molecular unit placed in an extremely weakly-bound cluster is readily excited, leading either to dissociation or to additional vibrotational excitation, with rise times of fs and energy transfer of eVs, again without the requirement that there be matching of potentials or masses between the cluster and molecule. The rapid disassembly of the cluster as it impacts the wall and the subsequent impact of the pre-shocked molecule with the wall presents a multi-step sequence that may make experimental interpretation of the results in terms of the mechanisms 
leading to molecular fragmentation extremely difficult without complementary simulations carried out by computer molecular dynamics.

\section{Acknowledgments}

The authors gratefully acknowledge stimulating conversations with Dr. Charles G. Stevens (LLNL). This work was performed in part under the auspices of the U.S. Department of Energy by the Lawrence Livermore National Laboratory under contract number W-7405-ENG-48 and in part under the auspices of the U.S. Office of Naval Research under contract number N00014-89-F-0082P01.

\section{Bibliography}

[1] A. M. Karo and J. R. Hardy, Int. J. Quantum Chem. XII, Suppl. 1, 333 (1977).

[2] J. R. Hardy and A. M. Karo, Proceedings of the International Conference on Lattice Dynamics, M. Balkanski, Ed. (Flammarion Press, Paris, 1978), p. 163.

[3] F. E. Walker and R. J. Wasley, Prop. and Expl. 1, 73 (1976).

[4] A. M. Karo, J. R. Hardy, and F. E. Walker, Progress in Aeronautics and Astronautics, Vol. 87: Shock Waves, Explosions and Detonations, J. R. Bowen et al., Eds. (AIAA, Inc., New York, New York, 1983), p. 9.

[5] A. M. Karo and J. R. Hardy, Proceedings of the NATO Advanced Study Institute on Fast Reactions in Energetic Systems, C. Capellos and R. F. Walker, Eds. (D. Reidel Publishing Co., 1981), p. 611.

[6] A. M. Karo, J. R. Hardy, and F. E. Walker, Progress in Aeronautics and Astronautics, Vol. 75 : Gasdynamics of Detonations and Explosions, J. R. Bowen et al., Eds. (AIAA, Inc., New York, New York, 1981), p. 209.

[7] A. M. Karo, J. R. Hardy, and F. E. Walker, Shock Waves in Condensed Matter (Menlo Park), W. J. Nellis et al., Eds. (AIP Conference Proceedings No. 78, 1982), p. 92.

[8] A. M. Karo, J. R. Hardy, T. M. DeBoni, and F. E. Walker, Progress in Aeronautics and Astronautics, Vol. 94: Dynamics of Shock Waves, Explosions, and Detonations, J. R. Bowen et al., Eds. (AIAA, Inc., New York, New York, 1984), p. 405.

[9] A. M. Karo, J. R. Hardy, and M. H. Mehlman, Proceedings of the 15th International Symposium on Shock Waves and Tubes, D. Bershader and R. Hanson, Eds. (Stanford University Press, California, 1986), p. 885.

[10] A. M. Karo and J. R. Hardy, Int. J. Quantum Chem., Quantum Chem. Symp. 20, 763 (1986).

[11] A. M. Karo and J. R. Hardy, J. de Physique, Coll. C4, 48, 235 (1987).

[12] J. R. Hardy and A. M. Karo, J. Mol. Structure (Theochem) 181, 353 ( 1988 ).

[13] J. D. Beckerle et al., J. Chem. Phys. 86, 7236 (1987).

[14] J. D. Beckerle et al., J. Chem. Phys. 91, 5756 (1988).

[15] E. Kolodney and A. Amirav, Chem. Phys. 82, 269 (1983).

[16] E. Kolodney et al., Chem. Phys. Lett. 145, 177 (1988).

[17] C.-Y. Kung et al., Chem. Phys. Lett. 145, 455 (1988).

[18] C. G. Stevens, private conversation.

[19] C. W. Gear, Numerical Initial Value Problems in Ordinary Differential Equations (Prentice-Hall, Englewood Cliffs, New Jersey, 1971), Chapter VII.

[20] A. M. Karo and J. R. Hardy, Fourier Transform Studies of Shock-Induced Energy Flux in Trapped Organic Molecules, (to be published).

[21] F. J. Zerilli and E. T. Toton, Phys. Rev. B29, 5891 (1984); D. D. Dlott and M. D. Fayer, J. Chem. Phys. 92, 3798 (1990).

[22] A. M. Karo, G. A. Weiss, and J. R. Hardy (unpublished results). 